\title{
Detección de Cryptosporidium spp. y otros parásitos zoonóticos entéricos en perros domiciliados de la Ciudad de México
}

\author{
Detection of Cryptosporidium spp. and other enteric zoonotic \\ parasites in pet dogs of Mexico City \\ I Martínez-Barbabosa ${ }^{a^{*}}$, M Gutiérrez ${ }^{\text {b }}$, LA Ruiz ${ }^{\mathbf{b}}$, AM Fernández ${ }^{\text {, }}$, \\ EM Gutiérrez ${ }^{\text {a }}$,JM Aguilara ${ }^{a}$, M Shea $^{c}$, E Gaona $^{d}$ \\ aÁrea de Ciencias Básicas, Departamento de Atención a la Salud,Universidad Autónoma Metropolitana-Xochimilco, México. \\ bDepartamento de Microbiología y Parasitología, Facultad de Medicina, Universidad Nacional Autónoma de México, México. \\ 'Departamento de Educación y Comunicación Universidad Autónoma. Metropolitana Xochimilco, México. \\ ${ }^{\text {d} D e p a r t a m e n t o ~ d e l ~ H o m b r e ~ y ~ s u ~ A m b i e n t e, ~ U n i v e r s i d a d ~ A u t o ́ n o m a ~ M e t r o p o l i t a n a, ~ X o c h i m i l c o, ~ M e ́ x i c o . ~}$
}

\section{SUMMARY}

\begin{abstract}
Zoonotic intestinal parasites infecting dogs are a public health problem in several countries. The aim of this study was to determine the presence of Cryptosporidium spp. and other zoonotic enteric infection parasites in dogs residing in Mexico City. One hundred and eighty three stools were analysed by faecal smear and stained with the Ziehl Neelsen modified method, and CPS were also performed by the Faust method. To define the association between variables the chi-square and Fisher's exact were applied. The results showed an infection level of $21,3 \%(n=39)$ with one or more parasites. The infection frequency and percentage of Cryptosporidium spp., T. canis and Ancylostomideos was 21 (11.5\%), 11 (6\%) and 7 (3.8\%), respectively. The infection level of Cryptosporidium spp. was significantly higher in long-haired breeds. T. canis was detected in 6\% of them and Ancylostomideos in 3.8\%. The association of Cryptosporidium spp., and T. canis was $\mathrm{P}<0.05$. The same association was observed in the delegation of Contreras, $\mathrm{P}<$ 0.012 and Tlalpan, $\mathrm{P}<0.0000$. The infection level of Cryptosporidium spp., and T. canis was significantly higher in young dogs than in adults $(\mathrm{P}<$ 0.016). The presence of Cryptosporidium spp. and geohelminths in pet dogs of Mexico City was confirmed. Living with infected animals carries a risk of acquiring zoonoses. Dogs cohabiting with immunocompromised owners, especially long-haired breeds, should be checked frequently for zoonotic intestinal parasites. The level of infection of Cryptosporidium spp., T. canis and Ancylostomideos is an indicator of soil contamination and the risk of acquiring these parasites.
\end{abstract}

Key words: Cryptosporidium, T. canis, Ancylostomideos, epidemiology, canine.

\section{RESUMEN}

Los parásitos zoonóticos intestinales que parasitan al perro constituyen un problema de salud pública en diferentes países. El objetivo del estudio fue determinar la presencia de infección por Cryptosporidium spp., y otros enteroparásitos zoonóticos en perros domiciliados de la Ciudad de México. Se analizaron 183 muestras de materia fecal mediante extendido fecal coloreado con Ziehl Neelsen modificada y se realizaron CPS por el método de Faust. Para definir la asociación entre variables se emplearon las pruebas de chi-cuadrado y exacta de Fisher. El nivel de infección alcanzó 21,3\% ( $\mathrm{n}=39$ ), con uno o más parásitos. La frecuencia y porcentaje de la infección por Cryptosporidium spp., T. canis y Ancylostomideos fue de 21 (11,5\%), 11 (6\%) y 7 (3,8\%), respectivamente. El nivel de infección de Cryptosporidium spp. fue significativamente mayor en razas de pelo largo. T. canis se detectó en $6 \%$ y Ancylostoma spp. en 3,8\%. La asociación de Cryptosporidium spp. y T. canis fue $\mathrm{P}<0,05$. Esta misma asociación se observó en la delegación de Contreras con $\mathrm{P}<0,012$ y en Tlalpan $\mathrm{P}<0,0000$. El nivel de infección de Cryptosporidium spp. y T. canis fue significativamente mayor en perros jóvenes que en adultos $(\mathrm{P}<0,016)$. Se concluye que se comprobó la presencia de Cryptosporidium spp. y geohelmintos en perros domiciliados de la Ciudad de México. La convivencia con animales parasitados es un riesgo de adquirir zoonosis. Los perros, especialmente de pelo largo, en hogares de personas inmunodeficientes, deben ser evaluados frecuentemente en búsqueda de parásitos intestinales zoonóticos. El nivel de infección de Cryptosporidium spp., T. canis y Ancylostomideos es un indicador de la contaminación del suelo y del riesgo de adquirir estos parásitos.

Palabras clave: Cryptosporidium, T. canis, Ancylostomideos, epidemiología, canino.

\section{INTRODUCCIÓN}

Los parásitos zoonóticos intestinales que infectan al perro constituyen un problema relevante de salud

Aceptado: 18.12.2014.

* Edificio H-009, Calzada del Hueso 1100, Colonia Villa Quietud, CP. 04960, México D. F.; imarti@correo.xoc.uam.mx pública en numerosos países, destacándose entre ellos Cryptosporidium spp. y los geohelmintos Toxocara canis y Ancylostoma caninum. En este estudio nos enfocamos con mayor énfasis a Cryptosporidium spp., considerados como parásitos emergentes asociados a severos cuadros diarreicos en niños, inmunodeficientes e inmunocompetentes (Fayer et al 1997).

El género Cryptosporidium (Tyzzer 1907) incluye protozoos intracelulares que producen la cryptosporidiosis, 
ubicados en el Phylum Apicomplexa, clase Sporozoasida, subclase Coccidiasina, orden Eucoccidiorida, suborden Eimeriorina, familia Cryptosporiidae (Fayer y Ungar 1986). La cryptosporidiosis del hombre y de otros mamíferos era poco conocida antes de 1976 cuando se reportaron los primeros casos humanos en personas con inmunocompromiso. Sin embargo, con la aparición del síndrome de inmunodeficiencia adquirida (SIDA) se demostró que C. parvum se comporta como un organismo oportunista causante de diarrea en inmunocomprometidos, en niños de 6 a 24 meses de edad y en adultos inmunocompetentes. Actualmente se ha demostrado que es una de las infecciones entéricas más frecuentes en humanos y animales, así como un problema de salud pública mundial (Joachin 2004). La cryptosporidiosis es considerada como una zoonosis emergente por el CDC (Center for Disease Control) de los Estados Unidos de América, debido a que puede diseminarse en poco tiempo a grandes grupos de población (Rojas 2012). La escasa especificidad de hospederos permite transmitirse indistintamente entre los mamíferos domésticos y el hombre, causando significativas tasas de morbilidad y mortalidad a nivel mundial (Fayer et al 1997). La presencia de Cryptosporidium spp. en personas con diarrea en países en vías de desarrollo se estima en aproximadamente el $10 \%$ y en países desarrollados de 1 a 3\% (Cheng et al 2002). Estudios realizados en perros de países desarrollados mencionan infecciones del 2 al 18,4\% (Scorza y Tangtrongsup 2010, Itho et al 2014).

El principal mecanismo de infección es la ingesta de ooquistes esporulados presentes en agua y alimentos contaminados con materia fecal de animales o humanos parasitados con Cryptosporidium spp. (Carmena 2007, Chalmers y Katzer 2013, Robertson y Chalmers 2013, Hlavsa et al 2014, Widerströnning et al 2014).

La infección ocurre por la ingestión de ooquistes esporulados que resisten el pH ácido del estómago. En el duodeno la presencia de condiciones reductoras, enzimas pancreáticas así como sales biliares, y si las condiciones del hospedero son adecuadas, de cada ooquiste emergen cuatro esporozoitos en forma de plátano que invaden rápidamente los enterocitos de la mucosa intestinal. Del extremo anterior del parásito surge una vacuola que se fusiona con la membrana del enterocito para formar una interfase hospedero-parásito, y queda el parásito en una vacuola parasitófora de localización intracelular, pero extracitoplasmática. En la vacuola parasitófora el parásito se reproduce mediante las fases asexuadas de merogonia y esquizogonia. En la primera el trofozoito sufre tres divisiones nucleares dando origen a 8 merozoitos dentro de un esquizonte de primera generación o meronte I inmaduro; al romperse la vacuola parasitófora los merozoitos liberados en la luz intestinal siguen dos caminos, unos invaden otras células epiteliales para formar nuevamente el meronte I, y otros infectan nuevos enterocitos donde realizan dos divisiones nucleares asexuadas para formar el meronte II o esquizonte de segunda generación que contiene 4 merozoitos que al liberarse invaden otros enterocitos; en este momento sufren diferenciación sexual, dando lugar al microgametocito y al macrogametocito. Los macrogametocitos se transforman en macrogametos y los microgametocitos en microgametos. La unión de estos gametos origina el huevo o cigoto que posteriormente se transforma en ooquiste para completar el ciclo biológico. Cada ooquiste tiene una pared gruesa (doble cubierta) y cuatro esporozoitos, mide de 2 a $6 \mu$, al excretarse con las heces ya es infectante y resistente al medio ambiente. Los ooquistes de $C$. parvum pueden sobrevivir durante 6 meses (Fayer et al 1988). El 20\% de los ooquistes producidos tienen pared delgada que al romperse tras su salida de la célula hospedera libera los esporozoitos e invaden nuevos enterocitos. De esta forma Cryptosporidium tiene tres ciclos autoinfectivos: por un lado la reinfección continua de los merozoitos (meronte I y meronte II), y por otro lado los esporozoitos derivados de los ooquistes de pared delgada originan las infecciones crónicas asintomáticas en ausencia de reinfección exógena (Bouzid et al 2013).

En el perro la cryptosporidiosis es causada por $C$. canis (Fayer et al 2001, Xiao et al 2007), C. meleagridis (Miller et al 2003), C. muris (Lupo et al 2008), y $C$. parvum (Lloyd y Smith 1997). La infección por estos parásitos se observa con mayor frecuencia en individuos jóvenes y generalmente cursa asintomática, pero cuando los síntomas se hacen aparentes los más frecuentes son fiebre y diarrea acuosa acompañada de moco de olor fétido, dolor abdominal, falta de apetito, regurgitación, pérdida de peso, mala calidad de la piel, letargo, apatía, intolerancia al ejercicio y debilidad. En animales inmunocompetentes la infección intestinal por lo general es asintomática. En inmunocomprometidos causa enteritis, con excreción de ooquistes en las heces durante dos semanas, pero pueden desarrollar infecciones del aparato respiratorio, hígado, vías biliares y páncreas. C. parvum se ha reportado ocasionalmente como causa de diarrea en cachorros y perros infectados simultáneamente con el virus del moquillo y otras inmunodeficiencias. Algunos perros pueden desarrollar una forma de cryptosporidiosis grave que amenaza su vida, como en el caso de pacientes humanos que cursan con síndrome de inmunodeficiencia adquirida (SIDA) (Barr y Bowman 2007).

En humanos Cryptosporidium produce un síndrome diarreico de intensidad y duración variable. En personas inmunocompetentes la cryptosporidiosis presenta cuadros de diarrea acuosa y voluminosa con moco, sin sangre y ausencia de leucocitos, o puede cursar asintomática. El cuadro clínico se autolimita en aproximadamente 12 días. En personas inmunocomprometidas especialmente con SIDA, la diarrea tiende a ser severa y persistente, se manifiesta por evacuaciones profusas y acuosas, de gran frecuencia y volumen, con absorción anormal de vitamina $\mathrm{B}_{12}$, y presencia de esteatorrea, acompañándose de fiebre, náusea, vómito, ataque al estado general e importante pérdida de peso con alto grado de morbilidad y mortalidad 
(Farthing 2000, Insulander et al 2013). Otro grupo de personas en riesgo de adquirir la infección lo constituyen las personas desnutridas, sobre todo los menores de dos años de edad en los que la diarrea es intensa y prolongada acentuándose la desnutrición; en casos graves produce desequilibrio hidroelectrolítico severo que puede conducir a la muerte del paciente. Se ha mencionado que muchas de las infecciones humanas se adquieren a partir de animales de compañía, o de granja, infectados (Bouzid et al 2013, Cacció et al 2013, Santín 2013).

La importancia de este estudio radica en el hecho de que en la Ciudad de México no se cuenta con datos precisos sobre la cantidad de animales de compañía existentes, y mucho menos del número de perros domiciliados parasitados. Por lo que el objetivo de este trabajo fue detectar la presencia de infección por Cryptosporidium spp. y otros enteroparásitos zoonóticos en perros domiciliados de la Ciudad de México.

\section{MATERIAL Y MÉTODOS}

\section{DISEÑO DEL ESTUDIO}

Entre mayo y julio de 2013 se realizó un muestreo exploratorio descriptivo de corte transversal para la búsqueda intencionada de ooquistes de Cryptosporidium spp. y de otros enteroparásitos zoonóticos en perros domiciliados en la Ciudad de México.

\section{ÁREA DE ESTUDIO}

La Ciudad de México cuenta con 8. 851.080 habitantes. Se localiza entre los paralelos $19^{\circ} 11^{\prime}$ y $20^{\circ} 11^{\prime}$ de latitud norte y entre los meridianos $98^{\circ} 11^{\prime}$ y $99^{\circ} 30^{\prime}$ al oeste del meridiano de Greenwich, a $2.240 \mathrm{msnm}$, clima templado húmedo, temperatura media anual de $16^{\circ} \mathrm{C}$. Está formada por 16 delegaciones políticas distribuidas en una superficie de $1.495 \mathrm{~km}^{2}$ (INEGI, 2010).

\section{UNIVERSO Y MUESTRAS}

El estudio incluyó el análisis de 183 muestras de materia fecal obtenida de perros de 20 razas; $113(61,7 \%)$ machos y $70(38,3 \%)$ hembras, con edades de 3 meses a 12 años, colectadas en 12 delegaciones políticas de la Ciudad de México. Las delegaciones incluidas en el estudio y el número de nuestras fueron: Álvaro Obregón (10), Benito. Juárez, (9), Coyoacán (27), Cuajimalpa (10), Cuauhtémoc (9), Iztacalco (11), Iztapalapa (36), Contreras (9), Miguel Hidalgo (9). Tláhuac (5), Tlalpan (38), Xochimilco (10).

\section{RECOLECCIÓN DE LAS MUESTRAS}

A cada uno de los propietarios de los animales se le explicó el procedimiento para la recolección de las heces y se les entregó un envase hermético rotulado con los datos del animal y zona de colecta. El envase contenía como fijador $50 \mathrm{~mL}$ de formol diluido en solución de cloruro de sodio isotónica al 0,85\%. Al momento de la entrega cada muestra fue homogenizada inmediatamente y colocada en una red fría para su traslado a la Facultad de Medicina de la Universidad Nacional Autónoma de México.

\section{EXÁMENES COPROLÓGICOS}

En el Laboratorio de Inmunoparasitología de la Facultad de Medicina de la UNAM, cada muestra se procesó individualmente. Inicialmente se realizó un extendido fecal directo teñido con la técnica de Ziehl Neelsen modificada (Henriksen y Ponhlenz 1981). Simultáneamente se efectuó examen coproparasitoscópico de flotación-concentración con sulfato de zinc 1.18 (Faust et al 1938); con el sobrenadante se realizaron preparaciones en fresco teñidas con lugol. La observación se realizó en microscopios de campo claro Carl Zeiss a 100, 400 y 1000 X. La identificación de las formas parasitarias se realizó de acuerdo a su afinidad tintoreal y morfología.

\section{ANÁLISIS ESTADÍSTICO}

La información del muestreo se organizó y codificó en una base de datos en SPSS versión 21.0 para Windows (SPSS Inc, Chicago IL, USA). La información obtenida de las variables como presencia de Cryptosporidium spp. y otros enteroparásitos, edad, raza, sexo y delegación, se usaron para construir relaciones de asociación, gráficas y tablas de contingencia para tener una descripción cuantitativa de la población estudiada mediante el muestreo. En la búsqueda de asociación entre variables se emplearon las pruebas estadísticas Chi cuadrado y exacta de Fisher con un nivel de significancia de $0,05 \%$.

\section{RESULTADOS}

La figura 1 muestra la distribución de las principales razas caninas incluidas en el estudio, el grupo denominado otras corresponde a las razas con menos del $5 \%$ de perros. En el cuadro 1 se presentan las áreas de colecta, número de muestras de materia fecal y la frecuencia de perros infectados con enteroparásitos, que fue de 39 , con un nivel de infección de 21,3\%. Las frecuencias de perros infectados y sus porcentajes de infección por Cryptosporidium spp., T. canis y Ancylostomideos fueron de 21 (11,5\%), 11 (6\%) y 7 (3,8\%), respectivamente. El nivel de infección de Cryptosporidium spp. resultó estadísticamente significativo en perros de raza de pelo largo: Pastor Alemán $\mathrm{P}<0,046$, Golden $\mathrm{P}<0,047$, Cocker $\mathrm{P}<0,05$ y en perros criollos $\mathrm{P}$ $<0,002$. En la figura 2 se observa que los animales más infectados con Cryptosporidium spp. corresponden a menores de dos años de edad. La asociación de Cryptosporidium spp. con T. canis y Ancylostomideos, en el mismo grupo de edad, se obtuvo $\mathrm{P}<0,016$. 


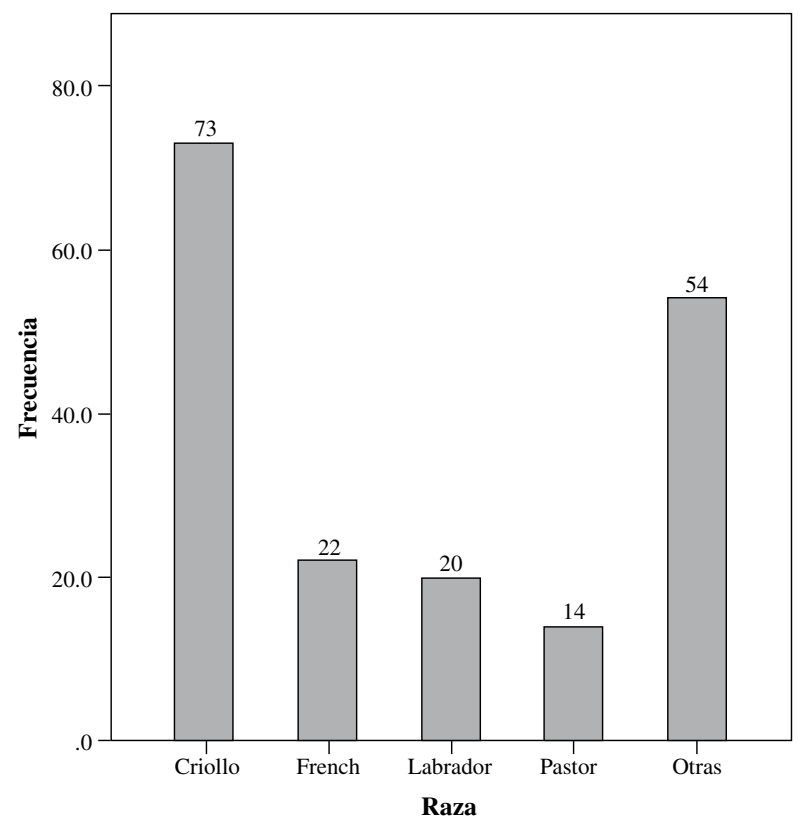

Otras: Incluye razas con menos del $5 \%$ de perros.

Others: breeds with a representation of less tan $5 \%$ in the sample.

Figura 1. Distribución de los 183 perros estudiados en diferentes razas.

Breed distribution of the 183 dogs analised.

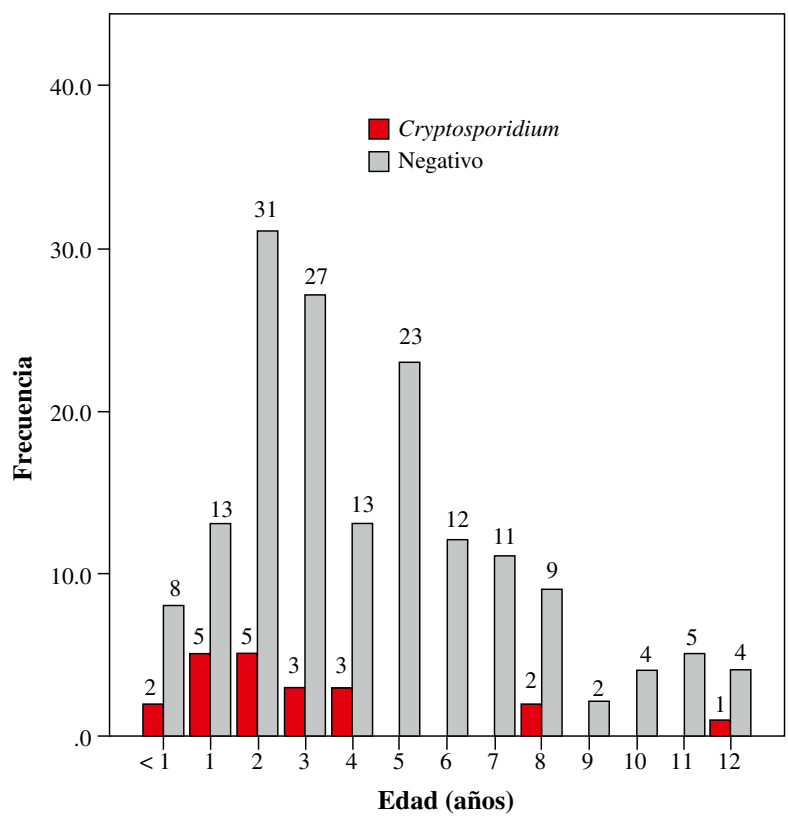

$* \mathrm{P}<0,016$, perros menores de 2 años de edad. $* \mathrm{P}<0.016$, dogs under 2 years of age.

Figura 2. Distribución por edad de los perros infectados con Cryptosporidium spp.*

The age distribution of the dogs infected by Cryptosporidium spp.*

Cuadro 1. Frecuencia de enteroparásitos zoonóticos en perros de las delegaciones estudiadas en la Ciudad de México. The frequency of the zoonotic parasites found by municipality of Mexico City.

\begin{tabular}{|c|c|c|c|c|}
\hline Delegación & Muestras & Criptosporidium & Ancylostomideos & T. canis \\
\hline A. Obregón & 10 & 0 & 1 & 0 \\
\hline B. Juárez & 9 & 3 & 1 & 0 \\
\hline Coyoacán & 27 & 3 & 0 & 0 \\
\hline Cuajimalpa & 10 & 0 & 0 & 0 \\
\hline Cuauhtémoc & 9 & 0 & 1 & 0 \\
\hline Iztacalco & 11 & 2 & 1 & 0 \\
\hline Iztapalapa & 36 & 1 & 1 & 3 \\
\hline Contreras & 9 & 3 & 0 & 3 \\
\hline Hidalgo & 9 & 0 & 0 & 1 \\
\hline Tláhuac & 5 & 1 & 0 & 1 \\
\hline Tlalpan & 38 & 6 & 1 & 2 \\
\hline Xochimilco & 10 & 2 & 1 & 1 \\
\hline Total & 183 & 21 & 7 & 11 \\
\hline
\end{tabular}

En el 61,9\% de los perros infectados se observó a Cryptosporidium spp. como parásito único y en el 38,1\% asociado a los geohelmintos T. canis y Ancylostomideos.

La asociación de Cryptosporidium spp. con T. canis y Ancylostomideos en el total de la muestra resultó significativo: $\mathrm{P}<0,0000$. Esta relación en los perros de las diferentes delegaciones también resultó significativa, con
$\mathrm{P}<0,05$, y en las de Iztacalco y Tlalpan, $\mathrm{P}<0,012$ y $\mathrm{P}<$ 0,0000 , respectivamente.

\section{DISCUSIÓN}

Se comprobó la presencia de Cryptosporidium spp. y de otros parásitos zoonóticos intestinales en perros 
domiciliados de la Ciudad de México. Los resultados sugieren que los perros de las delegaciones de Iztacalco y Tlalpan representaron mayor riesgo para transmitir las formas infectantes de Cryptosporidium spp., T. canis y Ancylostomideos a sus dueños.

El hallazgo de Cryptosporidium spp. en perros asintomáticos apoya la idea de que los cánidos infectados son una fuente potencial de infección para el humano (Lupo et al 2008). La infección con Cryptosporidium spp. en perros con dueño reportada en este estudio fue mayor a las obtenidas en estudios realizados en perros con características similares en ciudades como São Paulo, Brasil, 3,1\% (Katagiri y Oliveira-Sequeira 2008); Colorado, EE.UU., 3,8\% (Hackett y Lappin 2003), y es semejante a las notificadas en Zaragoza, España, 7,4\% (Causape et al 1996); Niágara, Canadá, 7,4\% (Shukla et al 2006); Praga, República Checa, 9,3\% (Dubna et al 2007); Osaka, Japón, 9,3\% (Abe et al 2002) y con el 8,7\% en los Países Bajos (Overgaauw et al 2009). Sin embargo, los datos de infección obtenidos en este trabajo fueron inferiores al $40 \%$ reportado en la ciudad de Campos dos Goytacases, Brasil (Ederil et al 2005).

En la discrepancia de los resultados anteriores influyen diversos factores como son las condiciones climáticas del lugar de estudio, las características del suelo, el grado de contaminación del mismo, los factores socioculturales y el procedimiento de laboratorio empleado.

En la cryptosporidiosis canina la principal fuente de infección la constituyen los animales jóvenes con diarrea. Sin embargo, los cánidos adultos también tienen un papel importante en la transmisión de estos microorganismos, porque como portadores asintomáticos eliminan constantemente un reducido número de ooquistes, pero suficientes para infectar a los neonatos (Fayer et al 2001). La morbilidad de la enfermedad se explica por el elevado número de ooquistes que evacuan los animales infectados y que desde este momento ya son infectantes. En el momento del estudio, el estado general de los perros y la consistencia de sus heces se consideraron normales, por lo que deducimos que la infección cursaba asintomática. De esta forma, la infección asintomática de los animales pasa inadvertida para sus dueños, y se convierten en fuente de infección para la familia y para el propio animal al contaminar el suelo con ooquistes esporulados infectantes.

El problema se agudiza aún más si tenemos en cuenta la concatenación de algunos factores biológicos y climáticos inherentes al parásito, como son la baja dosis de ooquistes infectantes requerida (10 ooquistes) para desarrollar la infección (De Graaf et al 1999). Otro aspecto característico es que su ciclo biológico pasa por tres fases reproductivas autoinfectantes, así como la producción de ooquistes de pared delgada que al exquistarse en el intestino del hospedero parasitado lo reinfectan con el consiguiente aumento en el número de parásitos, como es el caso de los terneros parasitados que llegan a eliminar diariamente hasta $2 \times 10^{9}$ ooquistes (De Graaf et al 1999), o el de perros infectados experimentalmente que llegan a eliminar 10.000 ooquistes por gramo de heces (Lloyd y Smith 1997). De esta forma la gravedad del cuadro clínico en estos individuos es severa y originan infecciones crónicas asintomáticas de gran importancia epidemiológica. El ooquiste es resistente a los cambios del medio ambiente, así como a los tratamientos convencionales de potabilización del agua y a la mayoría de los desinfectantes de uso doméstico, por lo que permanecen infectantes. En ambientes húmedos como el que se encuentra en los jardines públicos o intradomiciliarios los ooquistes de $C$. parvum pueden permanecer viables durante 2 a 6 meses (Fayer et al 1998). El clima templado favorece la transmisión de Cryptosporidium spp., el que es característico de las zonas sur y sureste de la Ciudad, donde se ubican las delegaciones Contreras, Milpa Alta, Tláhuac y Xochimilco en las que se encontró el mayor nivel de infección de parásitos zoonóticos $(53,8 \%)$, caracterizadas también por contar con zonas agrícolas, animales de traspatio y gran variedad de animales sinantrópicos que pudieron haber intervenido en la transmisión de Cryptosporidium spp. en la población estudiada (Fayer et al 2001, Overgaauw et al 2009, Cacció et al 2013).

En personas de alto riesgo como es el caso de individuos inmunocomprometidos o en edades extremas de la vida, es conveniente recomendarles evitar el contacto con el pelo y las heces de animales infectados, así como mejorar los hábitos higiénicos y el lavado escrupuloso de manos inmediatamente después de tocar el pelo y los desechos de sus perros (Overgaauw et al 2009).

La presencia de geohelmintos en los perros analizados constituye otro problema relevante de salud pública, como es el caso de T. canis y Ancylostomideos. Las larvas de $T$. canis afectan diversos órganos tanto en perros como en humanos, sin embargo, los parásitos adultos solamente afectan al perro. En el hombre al igual que en los cánidos, la forma infectante de T. canis es el huevo larvado, que al ser ingerido eclosiona y libera la larva del tercer estadio que puede migrar y producir granulomas en diferentes órganos y tejidos, cuyo grado de afectación estará en proporción directa al número de huevos larvados infectantes ingeridos (Schantz 1989). La forma clínica de la enfermedad conocida como larva migrante visceral puede incluir hepatomegalia, anorexia y malestar general en los pacientes. Los niños menores de 5 años de edad son los más afectados, y los principales factores de riesgo en esta población son la geofagia y el estrecho contacto con perros. La forma más grave de la enfermedad es la larva migrante ocular (LMO), ya que una sola larva puede causar ceguera unilateral (Keer-Muir 1994). El riesgo de convivir con perros domésticos parasitados con $T$. canis es tan grave, que recientemente se ha documentado un caso de toxocariasis ocular congénita en un recién nacido (Maffrand et al 2006). Cuando la larva filariforme de Ancylostomideos infecta al hombre por medio de la piel, le produce una lesión cutánea serpenteante de varios centímetros de longitud muy pruriginosa, resultado del labrado de un túnel 
en la epidermis conocida como larva migratoria cutánea, incapaz de invadir tejidos más profundos.

Consideramos que los niveles de infección de enteroparásitos obtenidos en los perros estudiados no deben subestimarse; a pesar de que el número de la muestra que comprendió el estudio no fue grande, sí es importante desde el punto de vista epidemiológico, debido a que se trata de animales domésticos asintomáticos pero parasitados, ya que eliminan constantemente al medio ambiente ooquistes y huevos de parásitos capaces de infectar a otros animales y al hombre mismo (De Graaf et al 1999; DuPont et al 1995).

Overgaauw et al (2009) detectaron en el pelo de perros domiciliados la presencia de huevos de Toxocara en diferente estadio de evolución y potencialmente infectantes para las personas mediante contacto directo. Un resultado estadísticamente significativo en este estudio fue el hallazgo de Cryptosporidium spp., T. canis y Ancylostomideos en los perros de razas de pelo largo como Pastor Alemán, Golden y Cocker. En este caso la manipulación del pelo de estos perros, sin las adecuadas precauciones higiénicas, podría facilitar la transmisión de Cryptosporidium spp. al dueño (Fayer et al 2001, Overgaauw et al 2009). Xiao et al (2007) reportaron la transmisión de Cryptosporidium canis de un perro familiar a dos hermanos en la ciudad de Lima, Perú.

En la transmisión de la infección se ha mencionado que la edad de los animales tiene un papel importante en la infección de los mismos, y que los jóvenes son los más susceptibles de adquirir la infección principalmente durante el primer año de vida. En este estudio la presencia de ooquistes de Cryptosporidium spp. resultó significativamente mayor en los animales menores de dos años de edad, coincidiendo con lo reportado por Lallo et al (2006).

En el presente estudio no se efectuó la identificación de la o las especies de Cryptosporidium en los perros estudiados. Tal identificación será necesaria en el futuro y en especial en aquellas compartidas con la especie humana, lo que permitirá estimar los factores de riesgo y medidas de prevención.

En la prevención de las enfermedades zoonóticas transmitidas por el perro es indispensable la participación directa de sus dueños con acciones como la desparasitación semestral, adecuado control de excretas y esterilización programada, esta última con el fin de contribuir a la disminución de la población canina callejera, ya que muchos cachorros son arrojados a la calle a temprana edad, acciones que de llegar a implementarse no dudamos que en poco tiempo se logrará tener un ambiente más sano para los habitantes de la Ciudad de México.

Por último, consideramos que los estudios relacionados con la presencia de parásitos intestinales en animales integrados a un núcleo familiar son indispensables para evaluar el verdadero impacto que estos pueden tener en la salud humana. Además, constituyen la base para recomendar medidas de control en programas de salud.
En poblaciones rurales el ganado vacuno parasitado con Cryptosporidium constituye la principal fuente de infección para el hombre. Sin embargo, en las grandes ciudades caracterizadas por tener una gran población canina (callejera y domiciliada) ignoramos la función que estos pueden tener en la dinámica de la trasmisión de esta zoonosis.

Se concluye que existe presencia de Cryptosporidium spp. en perros domiciliados asintomáticos principalmente en delegaciones del sur y sureste (Contreras, Tláhuac, Tlalpan y Xochimilco) de la Ciudad de México.

Los perros, en especial de pelo largo en hogares de personas inmunodeficientes, deben ser evaluados frecuentemente en búsqueda de parásitos intestinales zoonóticos.

La asociación de Cryptosporidium spp. con T. canis y Ancylostomideos es un indicador de la contaminación del suelo y del riesgo que representa para las personas y otros animales para la adquisición de parásitos.

La presencia de infección con Cryptosporidium spp. en perros domiciliados en la ciudad, requiere de más estudios acerca de la capacidad zoonótica de Cryptosporidium spp. en poblaciones humanas y animales, cuyos resultados serán de gran utilidad en el conocimiento y prevención de la cryptosporidiosis en México.

\section{REFERENCIAS}

Abe N, Y Sawano, K Yamada, I Kimata, M Iseki. 2002. Cryptosporidium infection in dogs in Osaka, Japan. Vet Parasitol 108, 185-193.

Barr SC, DD Bowman. 2007. Enfermedades infecciosas y parasitología en caninos y felinos. Inter-Médica S.A.I.C.I. Buenos Aires, Argentina, Pp 107-111.

Bouzid M, PR Hunter, RM Chalmers, KM Tyler. 2013. Cryptosporidium pathogenity and virulence. Clin Microbiol Rev 26, 115-134.

Cacció SM, AR Sannella, V Mariano, S Valentini, F Berti, F Tosini, E Pozio. 2013. A rare Cryptosporidium parvum genotype associated with infection of lambs and zoonotic transmission in Italy. Vet Parasitol 191,128-131

Carmena D, X Aguinagalde, C Zigorraga, JC Fernández-Crespo, JAOcio. 2007. Presence of Giardia cysts and Cryptosporidium oocysts in drinking water supplies in northern Spain. J Appl Microbiol 102, 619-629.

Causape AC, J Quilez, C Sánchez-Acevedo, E del Cacho. 1996. Prevalence of intestinal parasites, including Cryptosporidium parvum, in dogs in Zaragoza city, Spain. Vet Parasitol 67, 161-167.

Chalmers RM, F Katzer. 2013. Loofing for Cryptosporidium. The application of advances in detection and diagnosis. Trends Parasitol 29, 237-251.

Chen XM, JS Keithly, CV Paya, NF LaRusso. 2002. Cryptosporidiosis. $N$ Engl J Med 346, 1723-1731.

Current WL, LS García. 1991. Cryptosporidiosis. Clin Microbiol4, 325-358.

De Graaf DC, E Vanopdenbosch, LM Ortega-Mora, H Abbasi, JE Peeters. 1999. A review of the importance of cryptosporidiosis in farm animals. Int J Parasitol 29, 1269-1287.

Dubna S, I Langrova, J Napravnik, I JankovskaI, J Vadlejch, S Pekar, J Fechtner. 2007. The prevalence of intestinal parasites in dogs from Prague, rural areas, and shelters of the Czech Republic. Vet Parasitol 10, 120-128.

DuPont HL, CL Chappell, CR Sterling, PC Okhuysen, JB Rose, Jakubowski. 1995. The infectivity of Cryptosporidium parvum in healthy volunteers. N Engl J Med 332, 855-859.

Ederil BB, MF Rodrigues, CB Carvalho. 2005. Oocysts of the genus Cryptosporidium in domiciliated dogs from the city of Campos 
dos Goytacases, the State of Rio de Janeiro. Rev Bras Parasitol Vet 14, 129-131.

Farthing MJG. 2000. Clinical aspects of human cryptosporidiosis. Contrib Microb 6, 50-74.

Faust EC, JS D'Antoni, V Odom, MJ Miller, C Pares, E Sawitzl. 1938. A critical study of clinical laboratory techniques for the diagnosis of protozoans cysts and helminth eegs in feces. Am J Trop Medicin $18,169-193$.

Fayer R, BL Ungar. 1986. Cryptosporidium spp and Cryptosporidiosis. Microbiol Rev 50, 458-483.

Fayer R, CA Speer, JP Dubey. 1997. The general biology of Cryptosporidium. In: Cryptosporidium and cryptosporidiosis. $\mathrm{R}$ Fayer (Edit). Boca Raton, Florida. CR Press 1-41.

Fayer R, JM Trout, MC Jenkins. 1998. Infectivity of Cryptosporidium parvum oocysts stored in water at environmental temperatures. $J$ Parasitol 84, 1165-1169.

Fayer R, JM Trout, L Xiao, UM Morgan, AA Lai, JP Dubey. 2001. Cryptosporidium canis $\mathrm{n}$. sp. from domestic dogs. J Parasitol 87, 1415-1422.

Hackett T, MR Lappin. 2003. Prevalence of enteric pathogens in dogs of north-central Colorado. J Am Anim Hosp Assoc 39, 52-56.

Henriksen SA, JFL Ponhlenz. 1981. Staining of cryptosporidia by a modified Ziehl-Neelsen technique. Acta Vet Scand 22, 594-596.

Hlavsa MC, VA Roberts, AM Kahler, ED Hilbron, JT Wade, LC Backer, JS Yoder. 2014. Centros para el Control y Prevención de Enfermedades (CDC), Recreational water-associated disease outbreaks-United States, 2009-2010. MMWR Morb Mortal Wkly Rep 63, 6-10.

INEGI. Instituto Nacional de Geografía Estadística e Informática Centro de Población y Vivienda. 2010

Itoh N, Y Oohashi, M Ichikawa-Seki, T Itagari Y Ito, H Saeki, K Kanai, S Chikazawa, Y Hori, F Hoshi, S Higuchi. 2014. Molecular detection and characterization of Cryptosporidium species in householddogs, pet shop puppies, and dogs kept in a school of veterinay nursing in Japan. Vet Parasitol 1, 284-288.

Insulander M, C Silverlas, L Lebbas, L Karlsson, JG Mattsson, B Svenungsson. 2013. Molecular epidemiology and clinical manifestations of human cryptosporidiosis in Sweden. Epidemiol Infect 141, 1009-1020.

Joachin A. 2004. Human Cryptosporidiosis: An Update With Special Emphasis on the Situation in Europe. J Vet Med 51, 251-259.

Katagiri A, TC Oliveira-Sequeira. 2008. Prevalence of dog intestinal parasites and risk perception of zoonotic infection by dog owners in Sao Paulo State, Brazil. Zoonoses Public Health 55, 406-413.
Keer-Muir MG. 1994. Toxocara canis and human health. Br Med J 2, 296-297.

Lallo MA, EF Bondan. 2006. Prevalence of Cryptosporidium sp. In institutionalized dogs in the city of Sao Paulo Brazil. Rev Saude Publica 40, 120-125.

Lloyd S, J Smith. 1997. Pattern of Cryptosporidium parvum oocyst excretion by experimentally infected dogs. Int J Parasitol $27,799-801$.

Lupo PJ, RC Langer-Curry, M Robinson, PC Okhuysen, CI Chappell. 2008. Cryptosporidium muris in a Texas canine population. Am J Trop Med Hyg 78, 917-921.

Maffrand R, M Ávila-Vázquez, D Princich, P Alasia. 2006. Toxocariasis ocular congénita en un recién nacido prematuro. An Pediatr 64, 599-600.

Miller D, A Liggett, ZA Radi, LO Branch. 2003. Gastrointestinal cryptosporidiosis in a puppy. Vet Parasitol 115, 199-204.

Overgaauw PA, L van Zutphen, D Hoek, FO Yaya, J Roelfsema, E Pinelli, F van Knapen, LM Kortbeek. 2009. Zoonotic parasites in fecal samples and fur from dogs and cats in The Netherlands. Vet Parasitol 163, 115-122.

Robertson LJ, RM Chalmers. 2013. Foodborne cryptosporidiosis: is there really more in Nordic countries? Trens Parasitol 29, 3-9.

Rojas C. 2012. Cryptosporidium spp: Un parásito emergente asociado a diarrea. Rev Gastrohnup 4 (Supl. 1), S20-S24.

Santín M. 2013. Clinical and subclinical infections with Cryptosporidium in animals. NZ Vet J 61, 1-10.

Schantz PM. 1989. Toxocara larva migrans now. Am J Trop Med Hyg 4, suppl 21-34.

Scorza V, TS Angtrongsup. 2010. Update on the Diagnosis and Management of Cryptosporidium spp Infections in Dogs and Cats. Top Companion Anim Med 25, 163-169.

Shukla R, P Giraldo, A Kraliz, M Finnigan, AL Sánchez. 2006. Cryptosporidium spp. And other zoonotic enteric parasites in a sample of domestic dogs and cats in the Niagara region of Ontario. Can Vet J 47, 1179-1184.

Xiao L, VA Cama, L Cabrera, Y Ortega, J Pearson, RH Gilman. 2007. Possible transmission of Cryptosporidium canis among children and a dog in a household. J Clin Microbiol 45, 2014-2016.

Wiiderströnning M, C Schönning H Lija, M Lebbad, T Ljung, T Allestam, M Ferm, B Björkholm, A Hansen, J Hiltula, J Lanmark, M LÖfdahl, M Omberg, C Reuterwall, E Samuelsson, K Widgren, U Wallensten, J Lindh. 2014. Large outbreak of Cryptosporidium hominis infection transmitted through the public water supply, Sweden. Emerg Infect Dis 20, 581-589. 
